\title{
Particle dispersion in synthetic turbulent flows
}

\author{
R. Reigada \\ Departament de Química Física, Universitat de Barcelona, Avinguda Diagonal 647, Barcelona 08028, Spain \\ A. C. Martí \\ Instituto de Física, Facultad de Ciencias, Iguá 4225, 11400 Montevideo, Uruguay \\ I. M. Sokolov \\ Theoretical Polymer Physics, University of Freiburg, Hermann-Herder-Strasse 3, D-79104 Freiburg, Germany \\ F. Sagués \\ Departament de Química Física, Universitat de Barcelona, Avinguda Diagonal 647, Barcelona 08028, Spain
}

J. M. Sancho

Departament d'Estructura i Constituents de la Matéria, Universitat de Barcelona, Avinguda Diagonal 647, Barcelona 08028, Spain

(Received 12 April 2000)

\begin{abstract}
We study particle dispersion advected by a synthetic turbulent flow from a Lagrangian perspective and focus on the two-particle and cluster dispersion by the flow. It has been recently reported that Richardson's law for the two-particle dispersion can stem from different dispersion mechanisms, and can be dominated by either diffusive or ballistic events. The nature of the Richardson dispersion depends on the parameters of our flow and is discussed in terms of the values of a persistence parameter expressing the relative importance of the two above-mentioned mechanisms. We support this analysis by studying the distribution of interparticle distances, the relative velocity correlation functions, as well as the relative trajectories.
\end{abstract}

PACS number(s): 47.27.Qb, 47.27.Eq, 05.40.-a

\section{INTRODUCTION}

The diffusion of a passive scalar convected by a statistically homogeneous and isotropic turbulent flow is a problem of practical and fundamental interest in a great variety of contexts such as chemical reactions, mixing of fluids, and spreading of pollutants. Many important results on turbulent diffusion are formulated in Lagrangian coordinates (see, for example, Monin and Yaglom [1] and McComb [2]), where the coordinate frame is associated with a moving fluid element. Since no simple connections between the Lagrangian properties of the flow and the Eulerian properties of the velocity field (measured in a laboratory frame) can be formulated, much effort was invested into numerical modeling of flows with given Eulerian or Lagrangian characteristics.

Our approach is based on the use of a two-dimensional synthetic turbulent flow with prescribed statistical properties. In our simulations we first generate the flow in all the system and then let the particles move advectively and without inertia according to the velocity field. Our method of generation of this field is based on a parallel update procedure. Although the algorithm spends much time with updating the whole-lattice velocity field (in comparison with the normal schemes where the velocity field is calculated only at the particle positions), it is suitable for the calculation of manyparticle properties, i.e., for the discussion of cluster dispersion, distance probability distributions, and some other position-dependent quantities that are going to be examined in this paper.

Our main aim is to study the Lagrangian dispersion of particles advected by the previously mentioned synthetic tur- bulent flow, as described by the Richardson's law. Since the initial work of L.F. Richardson [3], a large amount of work has been done [4-8] to understand the dispersion processes that lead to this behavior. It has been recently shown $[9,10]$ that different dynamical mechanisms can lead to the same Richardson's law for the two-particle dispersion, so that such dispersion can be dominated by either diffusive or ballistic events. In this paper we are going to support this idea with the numerical results of particle dispersions under our synthetic turbulence.

The paper is organized as follows: in Sec. II we introduce the synthetic flow and the way we generate it. In Sec. III we focus on Richardson's law and discuss the statistical nature of the process underlying this strongly enhanced dispersion. We mainly investigate the effects of the flow parameters on the Richardson's behavior. Although the dispersion law is the same, the modification of the turbulence parameters makes the dispersion mechanism more diffusionlike or more ballistic, depending on the typical length, correlation time, and mean-squared velocity of the flow. In Sec. IV we enforce the previous ideas by looking at the trajectories, distribution of interparticle distances, and relative velocity correlation functions. Finally, Sec. V contains the main conclusions.

\section{STOCHASTIC VELOCITY FIELDS}

In this section we describe a numerical method to generate a statistically homogeneous, isotropic, and stationary two-dimensional velocity field, which could represent a "synthetic', or "kinematic' turbulent flow with zero mean and well-defined statistical properties. Our present paper is 
entirely implemented in a two-dimensional space, however it is worth noting that it can be generalized to three dimensions (3D) [11]. The two-dimensional space is chosen here for several reasons. First of all, it is chosen for the sake of simplicity of the numerical simulations. Second, because the two-dimensional turbulence has considerable interest of its own. Such interest is connected with the experimental results that are important both from the fundamental (see [12] or more recently Paret and Tabeling, [13]) and from an applied viewpoint (see for example, Brown and Smith, [14]).

The starting point of our two-dimensional simulations is a Langevin equation for a stream function $\eta(\mathbf{r}, t)$,

$$
\frac{\partial \eta(\mathbf{r}, t)}{\partial t}=\nu \boldsymbol{\nabla}^{2} \eta(\mathbf{r}, t)+Q\left[\lambda^{2} \boldsymbol{\nabla}^{2}\right] \boldsymbol{\nabla} \cdot \zeta(\mathbf{r}, t)
$$

where $\nu$ is the kinematic viscosity. $Q\left[\lambda^{2} \nabla^{2}\right]$ denotes an operator which controls the spatial correlations with a characteristic length $\lambda$ and $\zeta(\mathbf{r}, t)$ is a Gaussian white-noise field with zero mean value and whose covariance is given by

$$
\left\langle\zeta^{i}\left(\mathbf{r}_{1}, t_{1}\right) \zeta^{j}\left(\mathbf{r}_{2}, t_{2}\right)\right\rangle=2 \epsilon_{0} \nu \delta\left(t_{1}-t_{2}\right) \delta\left(\mathbf{r}_{1}-\mathbf{r}_{2}\right) \delta^{i j},
$$

where the intensity of the noise $\epsilon_{0}$ is a parameter of the simulations. The Langevin equation can be formally integrated to get the temporal evolution of the stream function. Turning to a Fourier-space we see that Eq. (1) corresponds to building up the field from the independent Fourier modes and in this sense parallels to the kinematic simulations following the ideas of Refs. [15-18]. Using Eq. (1) corresponds to the change from an intrinsic randomness (associated to the complex behavior resulting from the nonlinearity of the Navier-Stokes equation) into a system of independent Fourier modes coupled to an external noise with prescribed statistical characteristics.

The incompressible two-dimensional velocity field follows then as

$$
\mathbf{v}(\mathbf{r}, t)=\left(-\frac{\partial \eta(\mathbf{r}, t)}{\partial y}, \frac{\partial \eta(\mathbf{r}, t)}{\partial x}\right)
$$

The main kinematic characteristics of the stochastic velocity field is the velocity correlation function $C(r, s)$, which is defined as

$$
C_{i j}(r, s)=\left\langle v_{i}\left(\mathbf{r}_{1}, t\right) v_{j}\left(\mathbf{r}_{2}, t^{\prime}\right)\right\rangle .
$$

As a consequence of the homogeneity, isotropy, and stationarity of the flow, the correlation functions depend only on relative coordinates $r=\left|\mathbf{r}_{1}-\mathbf{r}_{2}\right|$ of two points and on the time difference $s=\left|t-t^{\prime}\right|$. More specifically, we will employ the radial correlation function defined by

$$
C(r, s)=\frac{1}{2}\left[C_{x x}(r, s)+C_{y y}(r, s)\right] .
$$

The physical parameters characterizing the homogeneous and isotropic turbulent flow are the following: the meansquare velocity $u_{0}$ (intensity) defined in a way that

$$
u_{0}^{2}=C(0,0)=\int_{0}^{\infty} E(k) d k
$$

where $E(k)$ is the energy spectrum of the flow, and the characteristic (integral) time and length scales

$$
\begin{aligned}
& t_{0}=\frac{1}{u_{0}^{2}} \int_{0}^{\infty} d s C(0, s), \\
& l_{0}=\frac{1}{u_{0}^{2}} \int_{0}^{\infty} d r C(r, 0) .
\end{aligned}
$$

These parameters can be obtained as functions of the input parameters, $\nu, \epsilon_{0}$, and $\lambda$, for each specific form of the energy spectrum. In particular the spectrum is directly related with the $Q$ operator. In what follows we consider the KármánObukhov (KO) spectrum [19,20], which was introduced to study Kolgomorov turbulence and parametrizes the $E(k)$ function in the following way:

$$
E(k) \propto k^{3}\left[1+\frac{k^{2}}{k_{0}^{2}}\right]^{-7 / 3}
$$

This spectrum follows the widely accepted KolmogorovObukhov power law $E(k) \sim k^{-5 / 3}$ for the inertial range $(k$ $>k_{0}$ ) of well-developed homogeneous and isotropic turbulent flow. According to this energy spectrum the choice of the $Q$ operator is [11]

$$
Q\left[\lambda^{2} \nabla^{2}\right]=\left(1-\lambda^{2} \nabla^{2}\right)^{-7 / 6},
$$

where $\lambda=(9 / 5)^{1 / 2} k_{0}^{-1}$. In this case we derive for $C(r, s)$ the following expression:

$$
C(r, s)=\frac{\epsilon_{0}}{4 \pi} \int_{0}^{\infty} d k k^{3} J_{0}(k r)\left(1+\lambda^{2} k^{2}\right)^{-7 / 3} \exp \left(-\nu k^{2} s\right) .
$$

Equation (10) shows that the lifetime of the Fourier components of our flow behaves according to $\tau \propto \kappa^{-2}$, a signature of a diffusive process supposed by a Langevin dynamics. Thus, the lifetime of a structure of size $L$ grows proportionally to $\tau \propto L^{2}$ (as typical, say for the turbulent velocity fields in the viscous range) and does not follow the Kolmogorov scaling supposing $\tau \propto L^{2 / 3}$. Therefore, the larger structures of our flow are more persistent that ones in real turbulence. However this is not essential for the discussion of the dispersion properties of the flow since, as demonstrated, they are the same as those that follow from Kolmogorov's universality class.

The results for the three basic physical parameters, $u_{0}^{2}, t_{0}$, and $l_{0}$, in terms of the simulation parameters, $\epsilon_{0}, \lambda$, and $\nu$, are then

$$
u_{0}^{2}=\frac{9 \epsilon_{0}}{32 \pi \lambda^{4}}
$$

$$
t_{0}=\frac{\lambda^{2}}{3 \nu}
$$

$$
l_{0}=\lambda \frac{\Gamma(1 / 2) \Gamma(5 / 6)}{2 \Gamma(1 / 3)} .
$$


A detailed presentation of the way the algorithm just proposed is implemented to simulate turbulent flows can be found in Ref. [11]. We note that the scheme corresponds to a parallel updating of the velocity vectors on the lattice and thus is extremely effective for simulation of many-particle processes, such as dispersion of initially dense particle clusters. The whole procedure is discretized in space using a square lattice of $N \times N$ points and unit spacing $\Delta$. Concerning the temporal evolution, the Langevin equation is integrated exactly in the spatial Fourier space. It is worth noting that the initial conditions for the stream function $\eta(\mathbf{r}, 0)$ can be chosen in such a way that the flow is in its statistical steady state from the beginning of the simulation.

Note that the reproduction of the correct time dependence of Eulerian velocity field, following from the Kolmogorov's universality assumption and describing correctly both the lifetime of the structure of the flow and their sweeping by the overall flow, is an unsolved problem [8]. On the other hand, the practically oriented simulations of the two-particle dispersion often start from essentially frozen flow structures, assuming that the temporal decorrelation of the particles' relative motion takes place because the pair as a whole is moving, due to a mean velocity, relative to an essentially frozen flow (as proposed by a Taylor hypothesis, see Sec. 21.4 of Ref. [3]). This assumption serves as a basis for successful numerical approaches [16,21], see Sec. 6.5.1 of Ref. [10] for discussion. Thus, in applications, the time-dependent turbulent flow is often mimicked either by sweeping a frozen array of eddies past the laboratory frame by some constant velocity [21] or by sweeping indefinitely persisting eddies by the overall (self-consistent) velocity field [22], all leading to reasonable results. The velocity field in our case belongs essentially to the same class.

In Ref. [9] it was shown that the properties of the particle's dispersion in flows in which $\tau \propto L^{\beta}$ with $\beta>2 / 3$, behave essentially similar to those of Kolmogorov flows. The corresponding result was proved numerically in Ref. [23], following the quasi-Lagrangian algorithm of Boffetta et al. in Ref. [24]. The Lagrangian decorrelation process is then connected not to Eulerian decorrelation, but to sweeping along open flow lines. The effective correlation time then scales according to $\tau_{s}(r) \propto r / v(r) \propto t^{2 / 3}$, and the effective value of $\beta$ stagnates at the Kolmogorov value of $\beta=2 / 3$. In such situations the Richardson's law stems mostly from rare and ballistically separated pairs. On the other hand, this does not mean that the properties of such dispersion does not depend on the temporal correlations in the flow: we address this question in detail in Sec. III A. Moreover, the properties of manyparticle dispersion in such flow will be addressed in Sec. IV, in hope that they are generic for chaotic two-dimensional flows with Kolmogorov spatial scaling.

\section{LAGRANGIAN DISPERSION AND RICHARDSON'S LAW}

One of the benefits of our kinematic simulations is to elucidate the Lagrangian (multipoint) properties of flows with given Eulerian statistics. Within this perspective, we focus on questions concerning turbulent dispersion. In particular, the Richardson's law, giving a superdiffusive behavior for the mean relative square distance of particles advected by a turbulent flow, has been extensively studied. This law concerning the two-particle dispersion, was obtained by Richardson [3], by summarizing results of various experiments on the diffusion of ashes in the atmosphere. Formulated in terms of the distance between two fluid elements $R(t)$, initially in close vicinity, this law states that

$$
\left\langle R^{2}(t)\right\rangle=\kappa t^{3} .
$$

The prefactor $\kappa$ is typically expressed as $\kappa=G \varepsilon$, where $\varepsilon$ is the energy dissipation rate of the turbulent flow and $G$ is a dimensionless constant referred in the literature as the Richardson constant. Note that Richardson's law is also known as "four-thirds law," since Eq. (12) can be obtained from the diffusion equation with the diffusion coefficient $D(R)$ depending on $R$ as $R^{4 / 3}$. This important law can be understood in the general frame of Kolmogorov scaling description of turbulence $[1,2]$. There is a considerable amount of experimental data on relative dispersion supporting this law and in fact its range of validity is believed to go beyond the inertial subrange.

Although in our kinematic simulations no energy transfer and dissipation take place, we could formally define the energy dissipation rate $\varepsilon$ based on the prescribed energy spectrum [2]. Without going into the detail, the expression for $\varepsilon$ can be expressed as [11]

$$
\varepsilon \equiv-\frac{d E}{d t}=\int_{0}^{k_{\max }} d k 2 \nu k^{2} E(k, t),
$$

where $k_{\max }$ represents the cutoff of the inertial range. In our approach, due to computational limitations, the largest wave number $k_{\max }$ depends on $N$ and $\Delta$.

More useful for our formal scheme is the formulation of the Richardson's prefactor in terms of the scaling properties of the flow. Well-developed turbulent flows show the Kolmogorov's scaling, in which the mean-squared relative velocity at two points separated by a distance $r$

$$
\left\langle v_{r}^{2}(r)\right\rangle=\left\langle\left(\mathbf{v}\left(\mathbf{r}^{\prime}, t\right)-\mathbf{v}\left(\mathbf{r}^{\prime}+\mathbf{r}, t\right)\right)^{2}\right\rangle
$$

behaves as $\left\langle v_{r}^{2}(r)\right\rangle=A r^{2 / 3}$, where $A=C_{L} \varepsilon^{2 / 3}$ with $C_{L}$ being a numerical factor (connected in real $3 d$, flows with the Kolmogorov's constant defining the spectrum). The value of $A$ in our simulations can be analytically estimated (see the Appendix) from the general expression for the velocity correlation function, Eq. (10), and finally reads

$$
\left\langle v_{r}^{2}(r)\right\rangle \approx 2.637 \frac{u_{0}^{2}}{l_{0}^{2 / 3}} r^{2 / 3} .
$$

This expression has been checked numerically with our KO synthetic flows.

On the other hand, the value of the prefactor $\kappa$ in the Richardson's law, Eq. (12), can be interpreted in terms of a separation velocity $\mathbf{v}_{\text {sep }}(\mathbf{r})$, defined through $d \mathbf{r} / d t=\mathbf{v}_{\text {sep }}(\mathbf{r})$. According to Eq. (12) the separation velocity scales in the same way as one of the mean-square relative velocity of the flow, but with a different prefactor: $\mathbf{v}_{\text {sep }}^{2}(\mathbf{r})=B r^{2 / 3}$. Actually, the prefactor $B$ is related to that one appearing in the Richardson's law via $B=\frac{9}{4} \kappa^{2 / 3}$. The difference between the 

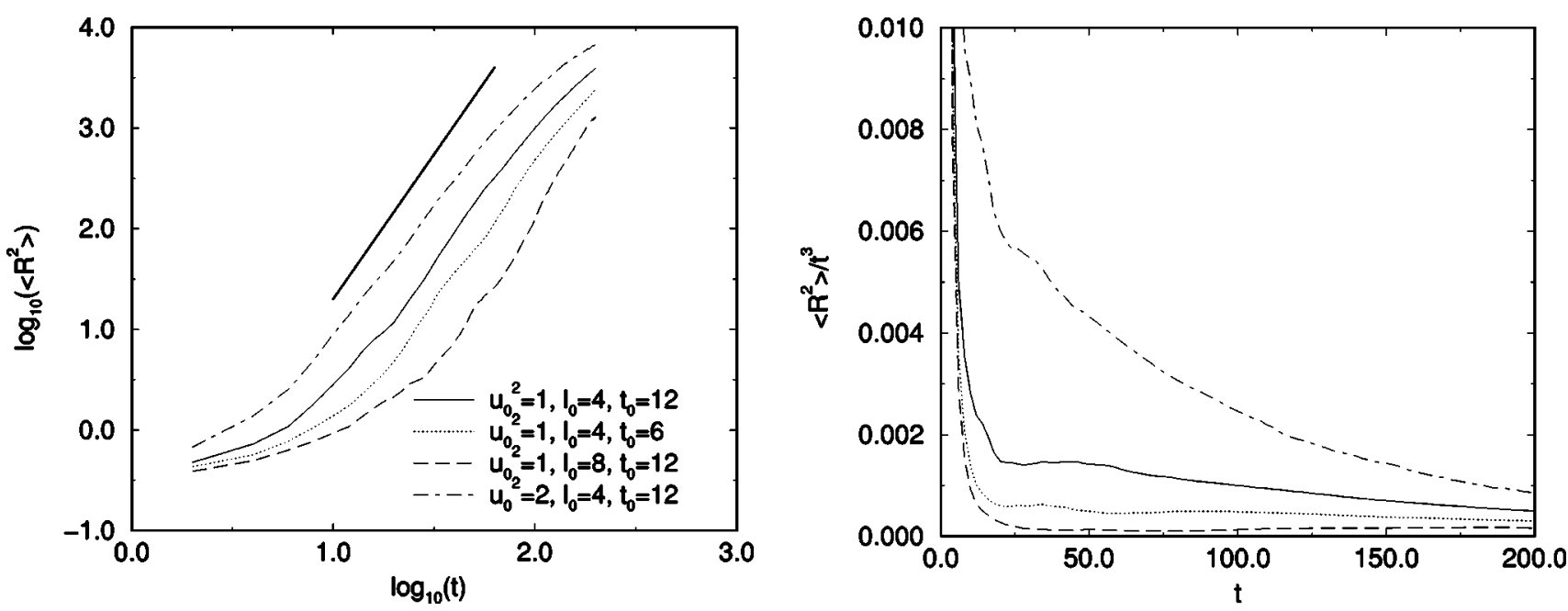

FIG. 1. $\left\langle R^{2}\right\rangle$ vs time for different sets of parameter values. Left panel: log-log representation. All the cases show reasonable agreement with the Richardson's law, $\left\langle R^{2}\right\rangle \sim t^{3}$. Right panel: $\left\langle R^{2}\right\rangle / t^{3}$ representation. Note that in all the figures in this paper no units are specified since the results of our simulations are dimensionless.

$\left\langle v_{r}^{2}(r)\right\rangle$ and $\mathbf{v}_{\text {sep }}^{2}(\mathbf{r})$ is due to the temporal decorrelation of the relative velocity of the particles, as will be discussed below.

\section{A. Numerical results}

The numerical simulation of the dispersion of passive particles by a synthetic two-dimensional flow is performed by integrating the equations of motion of these particles [25]

$$
\frac{d \mathbf{X}}{d t}=\mathbf{V}(\mathbf{X}, t),
$$

where $\mathbf{X}=(X, Y)$ is the position of the particle. The value of the velocity $\mathbf{V}(\mathbf{X})=\left(V^{x}(\mathbf{X}), V^{y}(\mathbf{X})\right)$ is interpolated using the bilinear form

$$
\begin{aligned}
V^{\alpha}(\mathbf{X})= & (1-\xi)(1-\mu) V_{p, q}^{\alpha}+(1-\xi) \mu V_{p, q+1}^{\alpha} \\
& +\xi(1-\mu) V_{p+1, q}^{\alpha}+\xi \mu V_{p+1, q+1}^{\alpha} .
\end{aligned}
$$

In this expression, $\alpha$ denotes the velocity's Cartesian component, $p=[X / \Delta]$ and $q=[Y / \Delta]$ give the coordinates of the grid's cell in which the point $\mathbf{X}$ is located, and the values $\xi$ $=\{X / \Delta\}$ and $\mu=\{Y / \Delta\}$ determine the relative position of a point within a cell. Here $[z]$ and $\{z\}$ denote the whole and fractional parts of $z$, respectively. $\mathbf{V}_{i, j}(t)$ stands for the discretized synthetic velocity field, Eq. (3), introduced in Sec. II.

We start from the set of an array of $M \times M$ particles with a closest interparticle separation fixed at 0.1 and placed within a square in the center of the system. We integrate Eq. (16) using a second-order Runge-Kutta method with a small step compared with the flow characteristic time $(\Delta t$ $\leqslant 0.1 t_{0}$ ). We average over 100 realizations of the flow in all the results in this work. Although we use periodic boundary conditions for the flow we do not want the particles to reach the boundaries of the system. When this happens we stop that realization and start another one. The final average for a variable at any given time only contains those realizations that still were valid at that time. Although we may loose some statistics at long times, we reduce much this effect by using large systems. When computing the two-particle distances $R$ we choose all the possible pairs, namely, we have $M^{2}\left(M^{2}-1\right) / 2$ pairs for each realization. Our systems are grids of $N=512$ with $\Delta=0.5$ and the discretization in time is $\Delta t=0.1$. All the parameters of the simulation (discretization values, size of the system, etc.) have been previously checked in order to reproduce the correct statistical properties of the flow and to ensure numerical stability.

The average relative distance is calculated according to

$$
R^{2}(t)=\frac{2}{M^{2}\left(M^{2}-1\right)} \sum_{i>j}\left\langle\left(\mathbf{X}_{i}(t)-\mathbf{X}_{j}(t)\right)^{2}\right\rangle .
$$

For intermediate times the Richardson's law, Eq. (12), applies and $\kappa$ is calculated from the plot of $\left\langle R^{2}(t)\right\rangle / t^{3}$ as a function of $t$, in the interval where this function is almost constant between the initial and asymptotic times. In this interval we fit a horizontal line and we could get the error in $\kappa$ from the difference between the maximum and the minimum value of the above-mentioned function during the plateau.

In order to study the dispersion features of the flow according to its statistical properties, we simulate some cases with different values of the flow parameters $u_{0}^{2}, l_{0}$, and $t_{0}$. Figure 1 shows the dispersion results for four of these cases where we can see the effect of the variation of each one of the flow parameters. We can calculate $B$ directly from the value of $\kappa$, and by using Eq. (15) for A, we compute the value of $\sqrt{B / A}$. Notice that $\sqrt{B / A}$ corresponds to the ratio between the separation velocity and the square root of the mean-squared relative velocity in Eq. (14). Within the model of Ref. [10] such a quantity is proportional to a persistence parameter $P_{s}$ of the flow. In Table I we have summarized these results for some cases, included those in Fig. 1.

When analyzing the results of Table I we realize, first of all, that all the values of $\sqrt{B / A}$ are much smaller than one. With respect to the effect of the turbulence parameters on this quantity, Table I shows that the longer the life of the eddies $\left(t_{0}\right)$, the larger the intensity of the flow $\left(u_{0}^{2}\right)$, and the 
TABLE I. Values of $\kappa, B, A$, and $\sqrt{B / A}$ for our numerical simulations with different turbulence parameters.

\begin{tabular}{lcccccc}
\hline \hline$l_{0}$ & $u_{0}^{2}$ & $t_{0}$ & $\kappa$ & $B$ & $A$ & $\sqrt{B / A}$ \\
\hline 8.0 & 1.0 & 8.0 & $6.60 \times 10^{-5}$ & 0.0036 & 0.659 & 0.0741 \\
8.0 & 1.0 & 12.0 & $1.61 \times 10^{-4}$ & 0.0066 & 0.659 & 0.1000 \\
8.0 & 1.0 & 20.0 & $3.78 \times 10^{-4}$ & 0.0117 & 0.659 & 0.1334 \\
8.0 & 1.0 & 24.0 & $4.62 \times 10^{-4}$ & 0.0134 & 0.659 & 0.1424 \\
8.0 & 4.0 & 8.0 & $2.28 \times 10^{-3}$ & 0.0389 & 2.637 & 0.1212 \\
8.0 & 4.0 & 12.0 & $3.52 \times 10^{-3}$ & 0.0520 & 2.637 & 0.1403 \\
8.0 & 4.0 & 16.0 & $5.28 \times 10^{-3}$ & 0.0682 & 2.637 & 0.1606 \\
8.0 & 4.0 & 20.0 & $6.33 \times 10^{-3}$ & 0.0769 & 2.637 & 0.1706 \\
8.0 & 4.0 & 24.0 & $7.92 \times 10^{-3}$ & 0.0893 & 2.637 & 0.1838 \\
8.0 & 6.0 & 8.0 & $6.20 \times 10^{-3}$ & 0.0759 & 3.955 & 0.1382 \\
8.0 & 6.0 & 12.0 & $8.71 \times 10^{-3}$ & 0.0952 & 3.955 & 0.1549 \\
4.0 & 1.0 & 12.0 & $1.43 \times 10^{-3}$ & 0.0285 & 1.046 & 0.1652 \\
6.0 & 1.0 & 12.0 & $3.91 \times 10^{-4}$ & 0.0120 & 0.798 & 0.1230 \\
10.0 & 1.0 & 12.0 & $1.02 \times 10^{-4}$ & 0.0049 & 0.568 & 0.0931 \\
4.0 & 1.0 & 6.0 & $5.45 \times 10^{-4}$ & 0.0150 & 1.046 & 0.1196 \\
4.0 & 2.0 & 12.0 & $5.60 \times 10^{-3}$ & 0.0709 & 2.092 & 0.1841 \\
\hline \hline
\end{tabular}

smaller are the eddies $\left(l_{0}\right)$, then the larger is the separation velocity. Figure 2 shows that the value of $B / A$ tends to follow a universal function of a dimensionless combination $u_{0} t_{0} / l_{0}$. Taking into account the fact that for some combination of parameters the Richardson's plateau in Fig. 1 (right) is rather short (which does not allow to determine the Richardson's constant with high accuracy), the quality of the scaling in Fig. 2 can be considered rather good. The combination $u_{0} t_{0} / l_{0}$ is similar in structure to a persistence parameter $P_{s}$ of the flow, introduced in Refs. [9] and [10] as a combination $P_{s}=v_{0} \tau_{0} / r_{0}$ of characteristic Lagrangian time, velocity, and length scales of the relative motion (see Sec. III B). This means that although no simple relation exists between these parameters and the Eulerian flow parameters $\left(u_{0}^{2}, t_{0}, l_{0}\right)$, the corresponding combinations are functionally dependent.

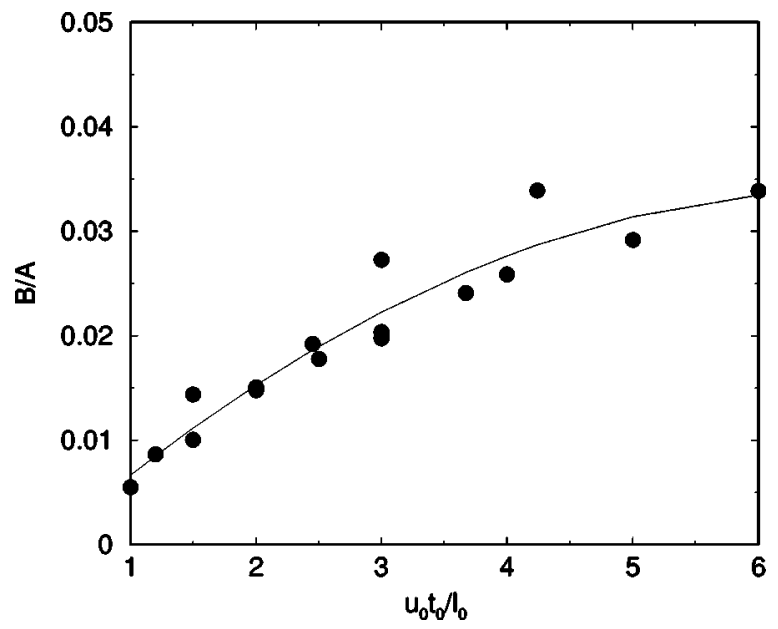

FIG. 2. Values of $B / A$ plotted against the dimensionless combination $u_{0} t_{0} / l_{0}$ for all the cases in Table $\mathrm{I}$. The full line is drawn as a guide for the eye.

\section{B. Theoretical background}

In order to understand the behavior described above, let us recall some results on well-developed turbulent flows showing Kolmogorov scaling. The two-time correlation function of the relative velocities, $\mathbf{v}_{r}(\mathbf{r}, t)=\left(\mathbf{v}\left(\mathbf{r}^{\prime}, t\right)-\mathbf{v}\left(\mathbf{r}^{\prime}\right.\right.$ $+\mathbf{r}, t)$ ), at points separated by the distance $r$, behaves as $[9,10]$

$$
\left\langle\mathbf{v}_{r}\left(\mathbf{r}, t_{1}\right) \mathbf{v}_{r}\left(\mathbf{r}, t_{2}\right)\right\rangle \propto\left\langle v_{r}^{2}(r)\right\rangle g\left[\left(t_{2}-t_{1}\right) / \tau(r)\right],
$$

where $\tau(r)$ is a distance-dependent correlation time, the $g$ function is defined so that $g(0)=1$, and $\left\langle v_{r}^{2}(r)\right\rangle$ was introduced in Eq. (14). Note that Eq. (19) applies to Lagrangian characteristics (pertinent to a situation where a coordinate frame is fixed on one of the particles of the pair).

The possible scenarios of the two-particle dispersion in a flow whose spatial scaling follows the Kolmogorov prescription were analyzed in Refs. [9,10], and [23]. Thus, one supposes

$$
\left\langle v_{r}^{2}(r)\right\rangle \propto v_{0}^{2}\left(\frac{r}{r_{0}}\right)^{\alpha}
$$

with $\alpha=2 / 3$. Let us assume in addition that

$$
\tau(r) \propto \tau_{0}\left(\frac{r}{r_{0}}\right)^{\beta}
$$

Then the Richardson law $\left\langle R^{2}(t)\right\rangle=\kappa t^{3}$ will hold asymptotically for the flows in which the value of $\beta \geqslant 2 / 3$. For a welldeveloped Kolmogorov case one would suppose $\beta=2 / 3$, in which case the properties of the flow would be described by a dimensionless number parameter $P_{s}=v_{0} \tau_{0} / r_{0}$, being a combination of characteristic Lagrangian time, velocity, and lengthscales of the relative motion. The parameter can be interpreted as a quotient of the mean-free path of the particles' relative motion and the actual distance, so that the small values of $P_{s}$ correspond to the dominance of the dif- 


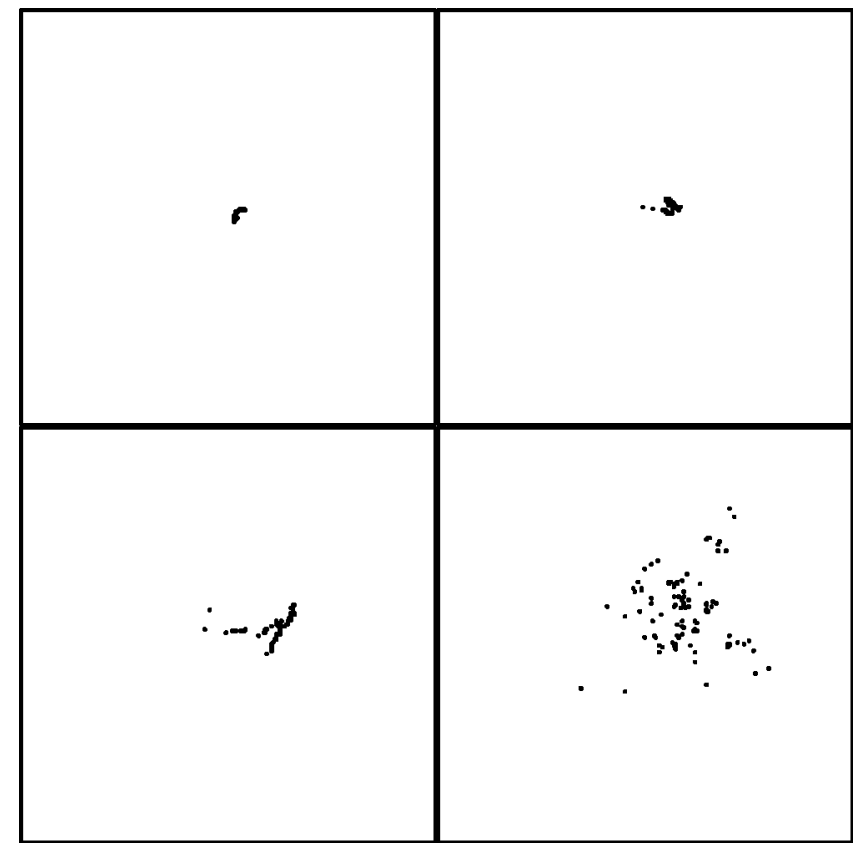

FIG. 3. Absolute positions of the $10 \times 10$ particles for the case with $u_{0}^{2}=1, l_{0}=4$, and $t_{0}=12$ shown in Fig. 1 . The snapshots correspond to times $t=20,40,60$, and 120 .

fusive transport, while the large $P_{s}$ values show that the transport is dominated by the ballistic events. For $P_{s}$ small, the value of $\sqrt{B / A}$ will be proportional to $P_{S}$.

The values of $\sqrt{B / A}$ listed in Table I make evident that the weight of the ballistic component of relative motion under all flow parameters considered is rather small, so that the Richardson's behavior corresponds to highly weigthed diffusive relative trajectories complemented with only a few ballistically separated pairs. As we proceed to show, this finding is compatible with a dispersion of the initial cluster in few smaller clusters. This idea is studied with more detail in Sec. IV A.

\section{FURTHER ANALYSIS OF SIMULATION RESULTS}

As anticipated, our simulation scheme allows us to extract additional information for particle dispersion apart from dispersion data concerning Richardson's law. This is going to be discussed in what follows.

\section{A. Analysis of particle trajectories}

Analyzing the trajectories of our cluster dispersion simulations help us to understand the significance of the flow persistence and the dispersion mechanisms leading to the Richardson's behavior. Since this behavior is characterized in terms of the interparticle distance variable, $R(t)$, we study both single and relative trajectories.

Let us consider a single realization of our numerical dispersion simulations, namely, a dense cluster of $10 \times 10$ particles in the middle of the system. As the time evolves, the typical picture of hydrodynamical mixing, consisting of the elongation and folding of the initial droplet, applies. As a result, an initially dense cluster is separated into several ones of similar density. This process is clearly seen in Fig. 3 for the positions of the 100 particles and in Fig. 4 for the relative

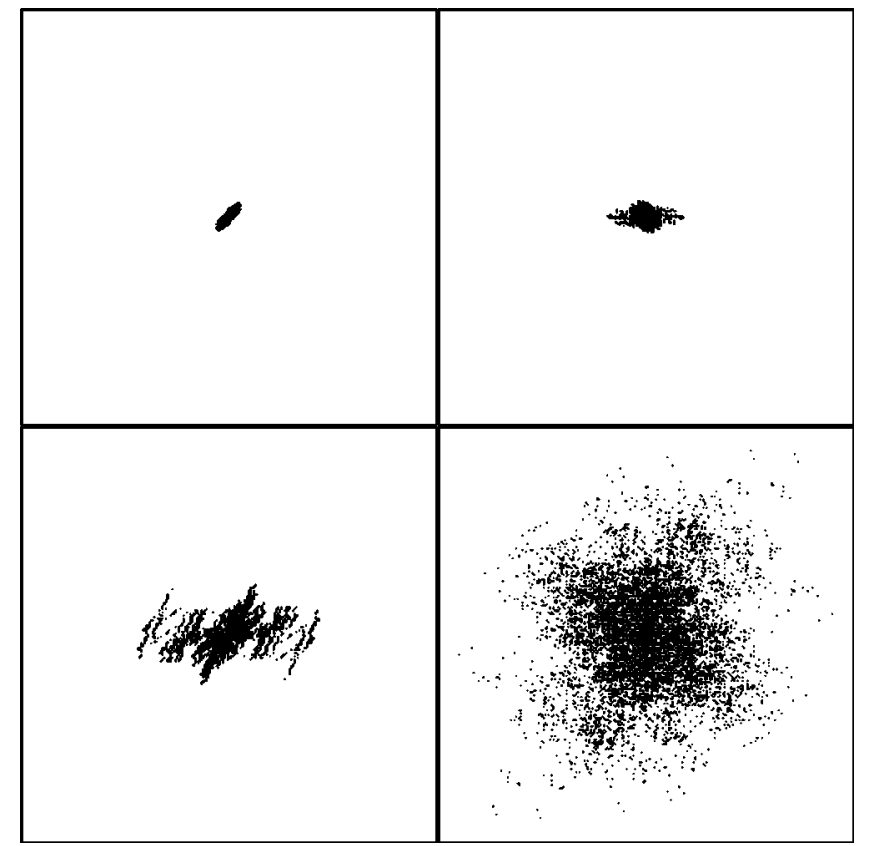

FIG. 4. Relative distances corresponding to the snapshots shown in Fig. 3.

distances of the 9900 possible pairs $[(i, j)$ and the symmetric pairs $(j, i)]$. The sequences in Figs. 3 and 4 correspond to times $t=20,40,60$, and 120 for the case with $u_{0}^{2}=1, l_{0}=4$, and $t_{0}=12$ shown in Fig. 1. At early times, close to the first snapshot, the particles move accordingly to the well-known elongation and folding mechanisms before the system enters the Richardson regime. Obviously, this early behavior is not captured by looking only at the first snapshot, since it corresponds to a single frame of the particles' evolution. The second and third pictures are taken during the Richardson's range. Notice how the system is still clusterized there. Moreover, in those times it is clearly seen how some ballistic events are evidenced in the relative representation. In spite of this, most of the relative positions are still concentrated in a central cluster corresponding to those pairs that have not been dispersed by the flow. The symmetric satellite groups (in the relative plots) can be associated with the separation of particles that are in different clusters, whereas the dense central cluster corresponds to the pairs that are traveling in the same cluster. The last snapshot stands for a very long time, when the particles are completely uncorrelated.

We can learn even more things by comparing this case (large- $t_{0}$ case) with, for instance, the same case with $t_{0}=6$ (small- $t_{0}$ case, also shown in Fig. 1). We show in Figs. 5 and 6 the positions and the relative distances, respectively, for this new case. By comparing both cases, we realize that the small- $t_{0}$ case shows a more clusterized way of dispersion, namely, the particles travel mostly together in fewer and denser clusters than in the large- $t_{0}$ case.

The behavior reported is coherent with the value of our effective persistence parameter $\sqrt{B / A}$. By looking at Table I we get that $\sqrt{B / A}=0.1652$ for the large- $t_{0}$ case and $\sqrt{B / A}$ $=0.1196$ for the small- $t_{0}$ case. Since the large- $t_{0}$ case has a larger value of $\sqrt{B / A}$ we found a larger amount of ballistic events than in the small- $t_{0}$ case. We can generalize this behavior by looking at the trajectories and relative trajectories 


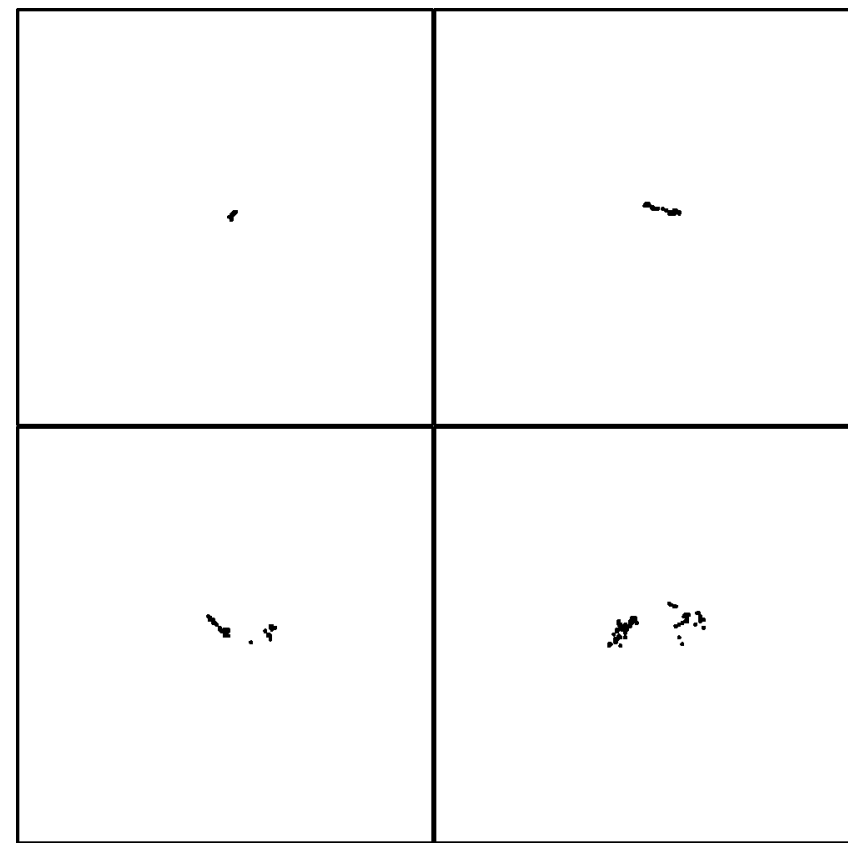

FIG. 5. Absolute positions of the $10 \times 10$ particles for the case with $u_{0}^{2}=1, l_{0}=4$, and $t_{0}=6$ shown in Fig. 1 . The snapshots correspond to times $t=20,40,60$, and 120 .

of the other cases in Table I. The cases with smaller $\sqrt{B / A}$ have fewer ballistic separations between particles and a larger number of diffusive and chaotic events than in the cases with larger $\sqrt{B / A}$.

In general, all the values of $\sqrt{B / A}$ for our flow (at least for the parameters shown in this work) are much smaller than 1 , and therefore the dispersion mechanism is mostly diffusive dominated. We have seen how most of the initially close pairs stay close to each other considerable times, performing similar motions even when $\left\langle R^{2}\right\rangle \sim t^{3}$ applies. In other words,

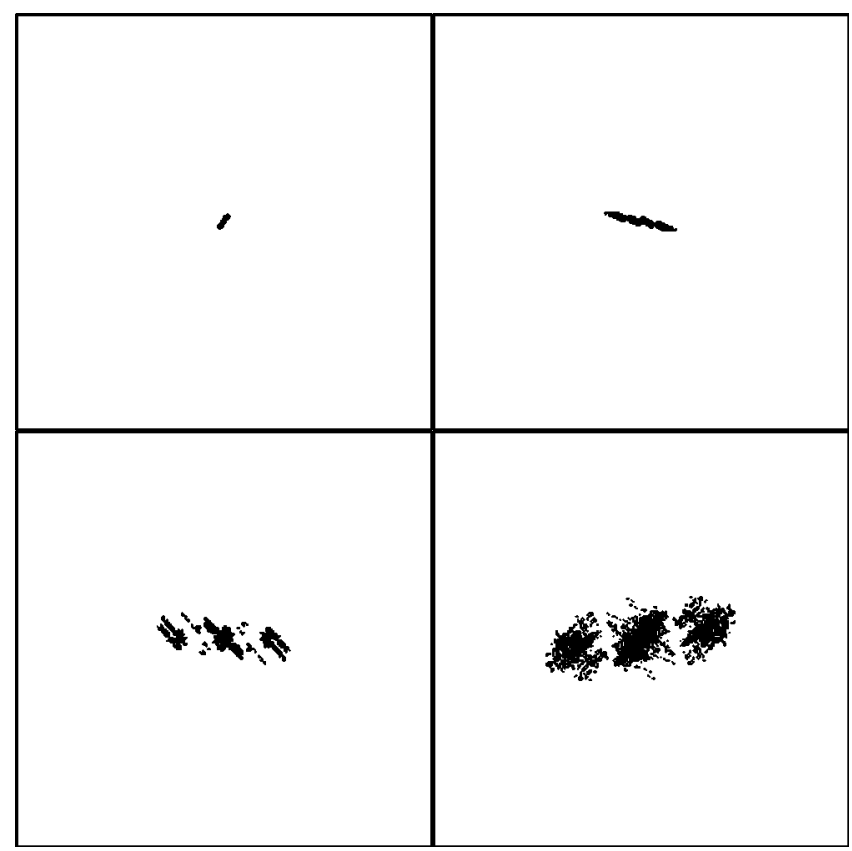

FIG. 6. Relative distances corresponding to the snapshots shown in Fig. 5.

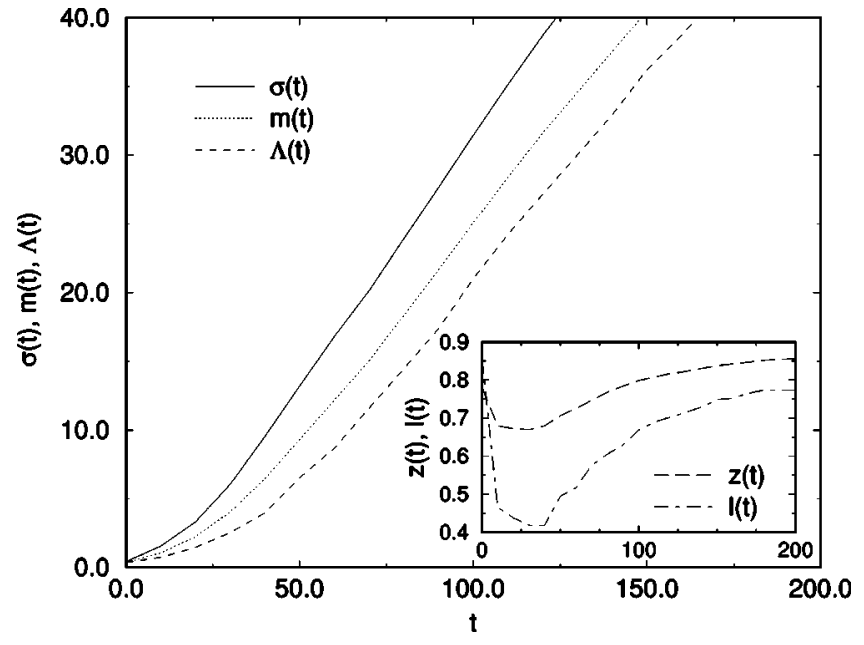

FIG. 7. Time evolution for the $\sigma(t), m(t)$, and $\Lambda(t)$ functions and the two relative moments $z$ and $l$. Results from the case with $u_{0}^{2}=1, l_{0}=4$, and $t_{0}=12$ in Fig. 1 .

Richardson's behavior for our flows comes to a large extent from rare ballistically separated pairs than from the typical separation of a pair within a cluster. This cluster nature of turbulent dispersion has been also seen for an experimental flow in Ref. [26].

\section{B. Distribution of interparticle distances}

To support this scenario we have computed the distribution of interparticle distances $P(R, t)$ at 10 different times for the case with $u_{0}^{2}=1, l_{0}=4$, and $t_{0}=12$ shown in Fig. 1 . We concentrated on the behavior of the median and the lowest moments of this distribution. Thus we calculate the square root of the second moment $\sigma(t) \equiv \sqrt{\left\langle R^{2}(t)\right\rangle}$, the first moment $m(t) \equiv\langle R(t)\rangle$, and the median $\Lambda(t)$ defined as the distance such that half of the pairs have a separation smaller that $\Lambda$.

The time evolution of $\sigma, m$, and $\Lambda$ is shown in Fig. 7. In this figure we also plot the relative moments $z \equiv m(t) / \sigma(t)$ and $l \equiv \Lambda(t) / \sigma(t)$. Both, the relative first moment $z$ and the relative median $l$ decay at the beginning, then they remain rather constant during the Richardson regime $[t \in(20$ $-50)]$ and finally they grow when the diffusive regime is reached. The behavior of both variables during the Richardson's range stems from the fact that most of the particles remain clusterized in the same number of clusters, or equivalently, that the distance between pairs is dominated by the separation between clusters already existing rather than by the formation of new ones. This supports the idea introduced in Sec. III and showed qualitatively in Sec. IV A. We can compare this behavior with the properties of the twodimensional Gaussian distribution and the Richardson's stretched-Gaussian law. Since both distributions scale with time, the values of $l$ and $z$ for them stay constant and are equal to $z=0.886$ and $l=0.832$, and $z=0.751$ and $l=0.565$ for the Gaussian and Richardson case, respectively [1]. Note in Fig. 7 that the value of $l$ in our case is even considerably lower than in the Richardson's case, which shows the very strong clusterization.

\section{Relative velocity correlation functions}

Another measure of the proportion of ballistically separated pairs can also be estimated by calculating the direction correlation functions of the relative velocities, 

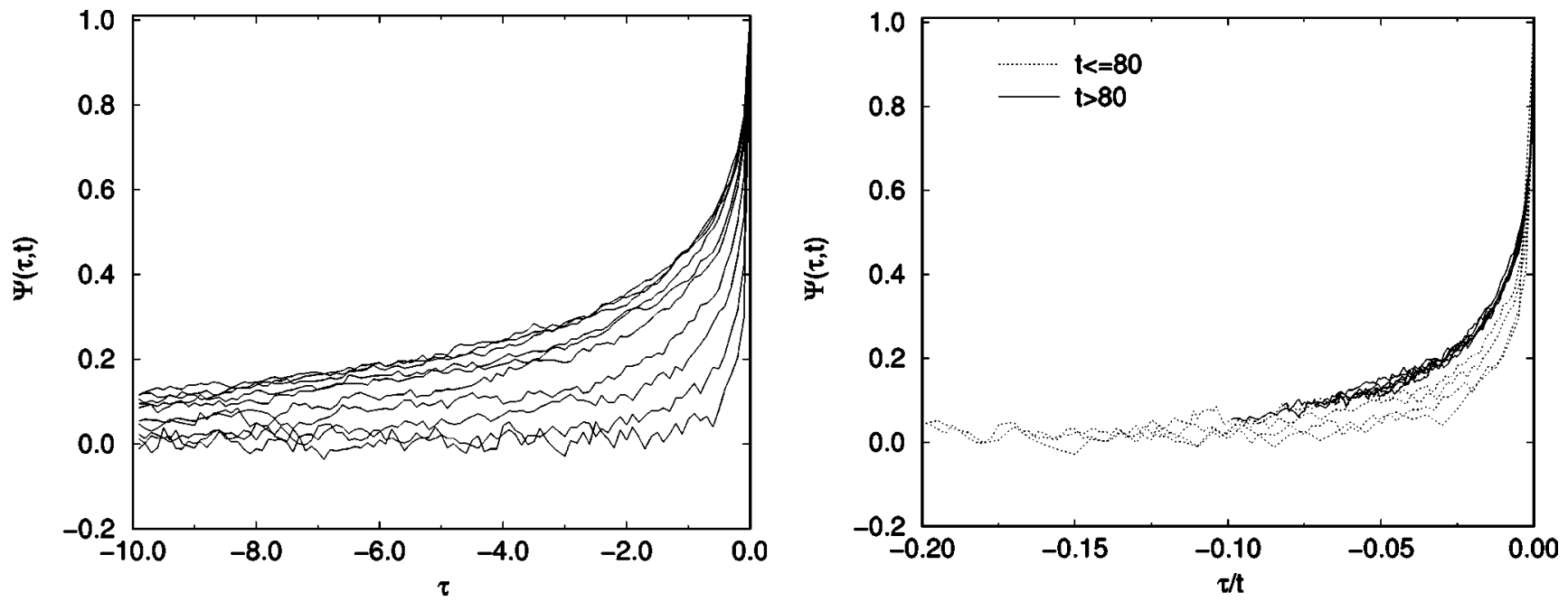

FIG. 8. Velocity correlation function $\Psi(\tau, t)$ for the case with $u_{0}^{2}=1, l_{0}=4$, and $t_{0}=6$ in Fig. 1 . Left panel: normal representation at times $t=20,40,60, \ldots, 200$ (from bottom to top). Right panel: scaled representation; notice how at times longer than 80 (solid lines) the functions scale quite well. We take the width of those scaled curves as the time memory of the flow.

$$
\Psi(t, \tau) \equiv\left\langle\mathbf{e}_{i j}(t) \mathbf{e}_{i j}(t+\tau)\right\rangle
$$

The brackets mean an average over pairs and statistical realizations of the flow, and $\mathbf{e}(t)$ is the unit vector of the direction of relative velocity of a pair $(i, j)$ at time $t$,

$$
\mathbf{e}_{i j}(t) \equiv \frac{\mathbf{v}_{i}(t)-\mathbf{v}_{j}(t)}{\left|\mathbf{v}_{i}(t)-\mathbf{v}_{j}(t)\right|} .
$$

The function $\Psi(t, \tau)$ is presented in Fig. 8 with $u_{0}^{2}=1$, $l_{0}=4$, and $t_{0}=6$ at times $t=20,40,60, \ldots, 200$ (from bottom to top) for the parameters of Fig. 5. For negative values of $\tau$ this function gives a quantitative measure of the memory in the relative motion. The characteristic time of this memory is given by the width of those correlation functions. From the left panel of Fig. 8 we infer that the memory time first grows until it stagnates at long times (when the particles become uncorrelated). In order to obtain a unique characterization of this time memory we scale $\Psi(t, \tau)$ with $\tau / t$, as shown in the right panel of Fig. 8. We can see how for short times (until $t=80$ ) the function does not scale at all, contrary to the behavior at longer times when the scaling is rather good. The width of this scaled representation gives us a quantitative characterization of the memory of the flow.

If we compare the width of the scaled correlation functions for different cases we can see how the systems with larger $\sqrt{B / A}$ (therefore, with more ballistic events), have a larger time memory. In Fig. 9 we plot the scaled correlation function for the cases in Fig. 1 for the time $t=120$, since all the cases scale at that time.

\section{CONCLUSIONS}

We have presented in this contribution a numerical study of the diffusion of passive scalars in synthetic turbulent flows. Making use of a practical algorithm to generate such flows, we have simulated the two-particles and cluster dispersion by a turbulent flow.

We have focused on the two different dispersion mechanisms (diffusive and ballistic) that lead to Richardson's be- havior. By defining our effective persistence parameter $\sqrt{B / A}$, we have quantified the proportion of either diffusive or ballistic events that coexist under the same $t^{3}$ law.

We have found that the larger are $t_{0}$ and $u_{0}^{2}$, and the smaller is $l_{0}$, the larger is $\sqrt{B / A}$, and therefore the larger number of pairs separate ballistically. We have also supported this idea by looking at the trajectories, the distribution of interparticle distances, and the relative velocity correlation functions. Moreover, the cluster nature of the dispersion under our turbulent flows is pointed out.

\section{ACKNOWLEDGMENTS}

Enlightening and valuable discussions with A. Blumen are gratefully acknowledged. R.R., F.S., and J.M.S. acknowledge the support of the Dirección General de Investigación Científica y Técnica (Spain) under Project Nos. PB961001 and PB96-0241, and by the Comissionat per Universitats i Recerca de la Generalitat de Catalunya. They also ac-

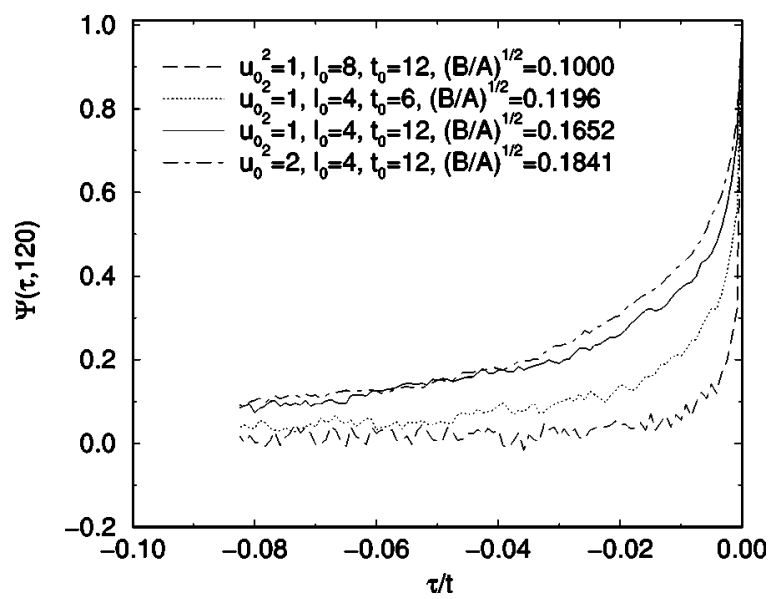

FIG. 9. Scaled velocity correlation functions $\Psi(\tau, t)$ for the four cases in Fig. 1. We compare the scaled functions at time $t=120$ since all the cases already show the scaling at that time. Notice how the time memory of each flow are consistent with their values of $\sqrt{B / A}$. 
knowledge computing support from Fundació Catalana per a la Recerca. I.M.S. acknowledges financial support received through the Fonds der Chemischen Industrie und through DFG. I.M.S. also acknowledges valuable discussions with J. Klafter and financial support received through GIF (GermanIsraeli Foundation). A.C.M. also acknowledges partial support from the CONICYT (Fondo Clemente Estable, 3046) and PEDECIBA (Uruguay).

\section{APPENDIX}

Starting from the definition of the mean-square relative velocity in Eq. (14) in terms of velocity correlation functions we have

$$
\left\langle v_{r}^{2}(r)\right\rangle=4\left(u_{0}^{2}-C(r, 0)\right) .
$$

If we rewrite Eq. (10) for the velocity correlation function in terms of the generalized hypergeometric series $F_{2}(a ; b, c ; d)$ [27] we obtain

$$
\begin{aligned}
C(r, 0)= & \frac{\epsilon_{0}}{4 \pi \lambda^{4}}\left[\frac{\Gamma(1 / 3)}{2 \Gamma(7 / 3)} F_{2}\left(2 ; 2 / 3,1 ; r^{2} / 4 \lambda^{2}\right)\right. \\
& \left.+\left(\frac{r}{\lambda}\right)^{2 / 3} \frac{\Gamma(-1 / 3)}{2^{5 / 3} \Gamma(4 / 3)} F_{2}\left(7 / 3 ; 4 / 3,4 / 3 ; r^{2} / 4 \lambda^{2}\right)\right] .
\end{aligned}
$$

For $r / \lambda$ much smaller than 1 ,

$$
C(r, 0)=\frac{\epsilon_{0}}{4 \pi \lambda^{4}}\left[\frac{\Gamma(1 / 3)}{2 \Gamma(7 / 3)}+\left(\frac{r}{\lambda}\right)^{2 / 3} \frac{\Gamma(-1 / 3)}{2^{5 / 3} \Gamma(4 / 3)}+o\left(r^{2} / \lambda^{2}\right)\right] .
$$

We know that $\Gamma(7 / 3)=\frac{4}{3} \Gamma(4 / 3)=\frac{4}{3} \frac{1}{3} \Gamma(1 / 3)$ and therefore

$$
C(r, 0)=\frac{\epsilon_{0}}{4 \pi \lambda^{4}}\left[\frac{9}{8}+\left(\frac{r}{\lambda}\right)^{2 / 3} \frac{\Gamma(-1 / 3)}{2^{5 / 3} \Gamma(4 / 3)}+o\left(r^{2} / \lambda^{2}\right)\right] .
$$

This expression can be reduced considering that $u_{0}^{2}$ $=9 \epsilon_{0} / 32 \pi \lambda^{4}$,

$$
C(r, 0)=u_{0}^{2}\left[1+\left(\frac{r}{\lambda}\right)^{2 / 3} \frac{4 \Gamma(-1 / 3)}{92^{2 / 3} \Gamma(4 / 3)}+o\left(r^{2} / \lambda^{2}\right)\right] .
$$

Including this expression into Eq. (A1) we obtain

$$
\left\langle v_{r}^{2}(r)\right\rangle=u_{0}^{2} \frac{16 \Gamma(-1 / 3)}{92^{2 / 3} \Gamma(4 / 3)}\left(\frac{r}{\lambda}\right)^{2 / 3}
$$

Finally, by replacing $\lambda$ by its dependence with $l_{0}$ we get

$$
\begin{aligned}
\left\langle v_{r}^{2}(r)\right\rangle & =\frac{u_{0}^{2}}{l_{0}^{2 / 3}} \frac{16 \Gamma(-1 / 3)}{92^{2 / 3} \Gamma(4 / 3)} \frac{\Gamma^{2 / 3}(1 / 2) \Gamma^{2 / 3}(5 / 6)}{2^{2 / 3} \Gamma^{2 / 3}(1 / 3)} r^{2 / 3} \\
& \approx 2.637 \frac{u_{0}^{2}}{l_{0}^{2 / 3}} r^{2 / 3} .
\end{aligned}
$$

[1] A.S. Monin and A.M. Yaglom, Statistical Fluid Mechanics (MIT, Cambridge, MA, 1975).

[2] W.D. Mc Comb, The Physics of Fluid Turbulence (Oxford University, Oxford, 1990).

[3] L.F. Richardson, Proc. R. Soc. London, Ser. A 110, 709 (1926).

[4] G.K. Batchelor, Q. J. R. Meteorol. Soc. 76, 133 (1950).

[5] G.K. Batchelor, Proc. Cambridge Philos. Soc. 48, 345 (1952).

[6] R.H. Kraichnan, Phys. Fluids 11, 945 (1968).

[7] H.G.E. Hentschel and I. Procaccia, Phys. Rev. A 29, 1461 (1984).

[8] A.J. Majda and P.R. Kramer, Phys. Rep. 314, 237 (1999).

[9] I.M. Sokolov, Phys. Rev. E 60, 5528 (1999).

[10] I.M. Sokolov, J. Klafter, and A. Blumen, Phys. Rev. E 61, 2717 (2000).

[11] A.C. Martí, J.M. Sancho, F. Sagués, and A. Careta, Phys. Fluids 9, 1078 (1997).

[12] U. Frisch, Turbulence: The Legacy of A. N. Kolmogorov (Cambridge University Press, New York, 1995).

[13] J. Paret and P. Tabeling, Phys. Rev. Lett. 79, 4162 (1997).
[14] M.G. Brown and K.B. Smith, J. Phys. Oceanogr. 20, 139 (1990).

[15] J.C.H. Fung, J.C.R. Hunt, U.A. Malik, and R.J. Perkins, J. Fluid Mech. 236, 281 (1992).

[16] J.C.H. Fung and J.C. Vassilicos, Phys. Rev. E 57, 1677 (1998).

[17] R.H. Kraichnan, Phys. Fluids 13, 22 (1970).

[18] R.H. Kraichnan, Phys. Rev. Lett. 78, 4922 (1997).

[19] A.M. Obukhov, C.R. Acad. Sci. USSR 32, 19 (1941).

[20] T.V. von Kárman, Proc. Natl. Acad. Sci. U.S.A. 34, 530 (1948).

[21] F.W. Elliot and A.J. Majda, Phys. Fluids 8, 1052 (1996).

[22] E.R. Abraham, Nature (London) 391, 577 (1998).

[23] I.M. Sokolov, e-print chao-dyn/9911006 (unpublished).

[24] G. Boffetta, A. Celani, A. Crisanti, and A. Vulpiani, Europhys. Lett. 42, 177 (1999).

[25] Preliminary results can be found in A.C. Martí, F. Sagués, and J.M. Sancho, Appl. Sci. Res. 59, 127 (1998).

[26] I.M. Sokolov and R. Reigada, Phys. Rev. E 59, 5412 (1999)).

[27] I.S. Gradshteyn and I.M. Ryzhik, Table of Integrals, Series and Products (Academic Press, New York, 1965). 